\title{
Public Communication in the MANAgemenT OF AN OUTBREAK OF INFECTIOUS DISEASE
}

Charles Watson

Health Department of Western Australia

\section{PART TWO: PLANNING AND COORDINATION}

D art One of this series covered issues involved in dealing with the mass media after the crisis has begun. The second and final part looks at what you can do to prepare yourself and your organisation for predictable and unpredictable crises, and examines strategic and logistic issues in coordinating the public communication response during a crisis.

\section{PLANNING}

Individual strategies for outbreak control must be carefully selected, well-timed and coordinated to be successful.

Planning may be done in the head of a single public health officer in some cases, but in others it requires the rapid formation of a team with a well-defined command structure and an adequate communication system.

\subsection{Prepare protocols in advance}

In any geographic area the possibility of serious infectious disease outbreaks can be predicted. In most cities in Australia outbreaks of whooping cough, measles, bacterial food poisoning and meningococcal meningitis must be anticipated. Preparation of protocols in advance for management of these and other outbreaks is a valuable investment. While such preparation is dependent on resources, protocols for management of particular outbreaks are so valuable it is worth examining documents from other States. Protocols should be treated as interim plans to be modified for local use.

A complete protocol will contain more than a plan and a series of instructions. It should also contain drafts of media releases, letters to school principals, good quality information sheets for GPs and other materials likely to be used. An excellent example is the package prepared by the British Meningitis Trust for dealing with outbreaks of meningococcal meningitis. The package contains brochures for parents, teachers and general practitioners, a $35 \mathrm{~mm}$ slide set and video cassette for community talks and a variety of other materials.

\section{COORDINATION}

Coordination of the communication strategies in an outbreak is often made difficult by the complexity of the situation, the spread of panic and the size of the problem. Coordination does not just happen - it has to be firmly put in place and actively supported.

\subsection{Appoint a coordinator and a planning committee} A single officer must be identified as the person responsible for coordinating all aspects of the response to the epidemic. In some cases the setting up of a small planning committee is useful. A reporting system must be established to ensure superiors (such as the regional director, the chief executive officer and the minister) are kept informed. The need to keep key officials informed is often overlooked in the midst of all the other urgent tasks but daily briefing memos are a vital part of crisis management. Some of the key people who need to be kept informed may be in other departments or organisations, such as those dealing with local government or education. Keeping them up to date ensures they have an opportunity to cooperate or provide active support rather than complain that no-one told them what was going on.

\subsection{Develop a public communication strategy and} nominate a single media contact.

The first step is to decide whether to say anything at all. But even if a decision is made to keep silent, preparation should be made for dealing with situations in which a reporter hears of the problem. Once a spokesperson has been identified, ensure that all media comment is channelled through this person. The public communication strategy should take into account the separate needs of the media, the general public and health professionals.

\subsection{The site visit}

When the first news of an outbreak arrives there is pressure to take immediate action. The information may be confused and incorrect and is almost certainly incomplete. It is sometimes possible to gather all necessary information by telephone, but in most situations there is no substitute for a personal visit by an experienced officer. The person visiting the site can get answers to questions no-one in head office had thought of asking, and can assess the capability of local infrastructure and the availability of resources for implementation of strategies such as immunisation. The visiting officer can give accurate advice to doctors and other health workers and can arrange a public meeting to discuss community concerns. The site visitor can establish a contact network of health workers, school principals, environmental health officers, reporters and others who might have a role in local management of the outbreak.

\subsection{Special communication services for major crises} The existing communication system may be adequate for small outbreaks, but in a major crisis special services will be required. In many crises the switchboard will be jammed and the command structure will collapse because normal telephone contact between health workers becomes impossible. Emergency arrangements must have been planned in advance. The most important element is to have one or more separate channels of communication available only to those managing the problem.

The easiest way for the coordinator to maintain essential contact is to have a mobile phone system to which limited people have access. A cellular phone means the coordinator is never out of contact and avoids the problem of jammed switchboards. Cellular phones can be rented.

Modern beepers with substantial capacity for messages can be used on their own or with a cellular phone. The best way is to use a beeper for incoming messages and keep the phone free for outgoing calls. Do not release the cellular phone number to more than one or two key individuals. Others can be given the number of the electronic message service and their calls can be returned when convenient.

Another useful method of indirect contact with the coordinator is by facsimile. Field officers providing information bulletins and reporters asking questions can contact the coordinator without jamming the command telephone system. However, fax machines are relatively slow and become ineffective in a crisis if many people have access to the number. One solution is to set up an emergency fax line in addition to the normal number and give the emergency number only to a limited number of people. 


\section{Public HeAlth ABSTRACTS}

$\mathrm{P}$ rofessor James S. Lawson, Professor and Head of the School of Health Services Management at the University of NSW, has prepared the following public health items from the literature.

\section{COUSIN OF HUMAN IMMUNODEFICIENCY VIRUS (HIV)}

Human T-Lymphotropic Virus Type 1 (HTLV-1) is known to be endemic in Japan, Africa, the Caribbean and Papua New Guinea and there are several reports of diseases associated with infection by HTLV-1 in Australia, indicating that the virus is also endemic in Australia, principally among Aboriginal groups. Like HIV, HTLV-1 has the ability to infect T-cells (cells related to the immune response) and has a very long 'incubation' period.

There are several diseases linked with HTLV-1. These include adult T-cell leukaemia, tropical spastic paraparesis - a chronic progressive disease of the nervous system - and inflammatory arthritis, plus a range of inflammatory conditions of the skin, the iris, retina and lungs. The virus is transmitted relatively inefficiently, primarily by breastfeeding, blood transfusion and sexual intercourse, and it penetrates populations considerably more slowly than does HIV. Fortunately, the number of carriers of the virus that goes on to develop disease is very low, somewhere between 1 in 1,000 and 1 in 3,000. Further cases of diseases associated with HTLV-1 can be expected in Australia. Diagnostic technology is now available using blood serum.

Heard RNS. H'TLV-1 in Australia Med J Aust 1993; 159-3-4.

\section{AIDS - DISCRIMINATION A PUBLIC HEALTH HAZARD}

In Central Africa 70 per cent of hospital beds are now occupied by patients with HIV-related diseases, and the number of AIDS-associated orphans increases daily. In the Middle East, the United States, the former Yugoslavia as well as Africa and Asia, human rights has become intimately linked with the AIDS epidemic. Discrimination against various groups has become a public health issue. For example, why should drug users in Bangkok with HIV be able to hold down steady jobs and be part of mainstream society, whereas their counterparts in the USA who seek clean syringes are promptly imprisoned? Discrimination against women is a prominent feature in every aspect of the AIDS epidemic. Their vulnerability in many societies a condom often costs more than a prostitute - is often encouraged in political manipulations of religious practices. AIDS has become far more than an epidemic.

Editorial: Ticket to dignity beyond a brick wall. Lancet 1993; 341:1625-1626.

\section{Public communication}

\section{$\checkmark$ Continued from page 99}

In a major crisis there will be hundreds or thousands of telephone inquiries from members of the public. Dealing with these inquiries is an enormous challenge. The first strategies are those which expand the capacity of the existing system - more switchboard operators, more officers to answer inquiries, and allocation of more telephone extensions to key areas. Many health departments have the capacity to set up a phone inquiry room with dedicated lines in an emergency and such facilities are also available for rental from Telecom.

The best way to deal with the public need for information is to provide what they want through other channels as soon as possible and to provide regular updates. This takes the pressure off the telephone system. The usual way to do this is with a media release and a news conference. Paid advertising can be a worthwhile alternative. The public need for information can also be satisfied by directing them to health workers such as general practitioners - as long as they have been adequately briefed.

An alternative which may be combined with paid or unpaid advertising is to publicise the number of a recorded message service, which can reduce the burden of personal telephone inquiries and also measure of public interest in the crisis.

General practitioners, pharmacists, hospital staff and other community-based health workers can satisfy public inquiries if they are adequately briefed. Their job is much easier if they are provided with information brochures to give to the public. An effective way to get information to doctors is to ask pathology laboratory services to attach a bulletin (supplied in bulk by the health authority) to pathology results being sent to the doctors. Community pharmacists can be supplied with bulletins or brochures through daily deliveries made by wholesalers. Health professionals such as GPs can be informed through media releases which also give information to the public.

A readable brochure can be of enormous value in informing the community about how to prevent further spread in an outbreak and in giving reassurance. Brochures can be distributed through GPs, pharmacies, hospitals and health insurance offices. They can be posted from the health department on request and their availability advertised. In outlying areas without radio and television, posters can be used effectively to give information on immunisation services or preventive behaviours.

Electronic mail is useful because of its speed and ability to contact large networks. An advantage of electronic mail is the ability to transfer documents in electronic form so they can be edited for local use without retyping.

\section{REVIEW}

When the crisis has ended, call a meeting of all major players to review the events. A critical review can be very valuable, not only in working out how to manage the same problem in the future, but in educating all those involved in the principles and practice of crisis management. The lessons learned can be applied to other problems you will face in the future. In many cases a review will result in organisational or equipment changes such as the installation of new phone lines or the purchase of cellular phones.

The review is also an important opportunity to acknowledge participants for the job they have done. Take notes during the meeting and circulate a summary to participants. Make sure the actions identified in the review are carried out. 